\title{
MAGNETORESISTANCE OF IODINE-DOPED POLYACETYLENE AT LOW TEMPERATURES
}

\section{E. Ettlinger and $W$. Schoepe}

Fakultät für Physik, Universität Regensburg, D-8400 Regensburg, W.Germany

$$
\text { M. Monkenbusch and G. Wieners }
$$

Institut für makromolekulare Chemie, Universität Freiburg, D-7800 Freiburg, W.Germany

(Received 29 August 1983 by E. Burstein)

We have measured the magnetoresistance of metallic polyacetylene at temperatures $\mathrm{T}$ between $4.2 \mathrm{~K}$ and $0.3 \mathrm{~K}$ and in fields $\mathrm{B}$ up to $3.4 \mathrm{Tesla}$ both parallel and perpendicular to the average current flow. We found an exclusively positive magnetoresistance scaling in low fields as $\mathrm{B}^{2} / \mathrm{T}^{2}$ for $\mathrm{T}<1 \mathrm{~K}$ and as $\mathrm{B}^{2} / \mathrm{T}^{3}$ for $\mathrm{T}>2 \mathrm{~K}$. At high doping levels the magnetoresistance is small and saturates in high fields at low temperatures. These results are independent of the particular geometry used and will be discussed in terms of the magnetic field effect on the hopping conductivity.

One of the intriguing properties of polyacetylene is its virtually metallic electrical conductivity when doped with suitable acceptors like iodine. In spite of a wealth of information that has been obtained about this fascinating organic conductor ${ }^{1}$ a detailed understanding of the electrical transport mechanism is still lacking, mostly because of the complicated morphology and the inhomogeneities associated with it.

In our present work we have measured the conductivity of iodine-doped polyacetylene $\left(\mathrm{CHI}_{\mathrm{y}}\right)_{\mathrm{x}}$ in the temperature range between $4.2 \mathrm{~K}$ and $0.33 \mathrm{k}$. We mostly studied samples having large iodine concentrations $(0.15 \leqslant y \leqslant 0.32)$, i.e. the metallic regime. Magnetic fields up to 3.4 Tesla were employed in both longitudinal and transverse geometries, including the Corbino arrangement. In all of our samples we found a positive magnetoresistance (MR) becoming very large at low temperatures. The more conducting samples had a smaller MR which saturated at low temperatures, irrespective of the particular geometry. Our results are thus different from those obtained earlier by Kwak et al. ${ }^{2}$ and Gould et $a 1 .{ }^{3}$ on $\mathrm{AsF}_{5}$ doped $(\mathrm{CH})_{\mathrm{X}}$ and by Röss et a1. ${ }^{4}$ who find a negative MR at $4.2 \mathrm{~K}$ in very metallic samples. Obviously, the detailed form and even the sign of the MR depends on how metallic the samples are prepared.

We used samples which were polymerized either according to Luttinger ${ }^{5}$ or Shirakawa ${ }^{6}$. Treating a $0.6 \mathrm{~mm}$ thick layer of Luttinger catalyst solution containing $1 \mathrm{mg}$ cobalt nitrate and $3 \mathrm{mg}$ sodium boronhydride per $\mathrm{ml}$ ethanol at $-78^{\circ} \mathrm{C}$ with acetylene gas results in a sponge like red polymer layer that was washed twice with ethanol and dried under vacuum. The resulting shiny film was about $40 \mu \mathrm{m}$ thick and weighed $3 \mathrm{mg} / \mathrm{cm}^{2}$. Alternatively, the wall of a $20 \mathrm{~cm}$ long round bottomed, evacuated flask of $5 \mathrm{~cm}$ diameter was wettet at $-78^{\circ} \mathrm{C}$ with a degassed Shirakawa catalyst solution containing $3.0 \mathrm{~m} 1$ aluminiuntriethyl and $1.9 \mathrm{ml}$ titanium tetrabutoxide in $20 \mathrm{ml}$ toluene, aged for one hour. Allowing acetylene gas to flow immediately into the flask, a film was formed, which, after removal of the unreacted catalyst solution, was washed 15 times with dry pentane and then dried. The resulting $\mathrm{film}$ was $80 \mu \mathrm{m}$ thick and weighed $2 \mathrm{mg} / \mathrm{cm}^{2}$. Both types of samples were stored under vacuum on dry ice.

Doping was performed by repeatedly exposing the samples to iodine vapor and consecutive vacuum pumping at room temperature. Iodine content of the samples was determined by weighing before and after doping. We did not find any differences between the Luttinger and Shirakawa samples, except for the observation that the latter ones could be doped more easily to higher levels. Only for this reason the data presented here are all from Shirakawa samples.

The samples were cooled in a ${ }^{3}$ He cryostat. In order to have good thermal contact the samples were immersed into the liquid ${ }^{3} \mathrm{He}$. The resistance was measured in a four-terminal method either with a high resolution dc voltmeter or with a low frequency ac bridge and a lock-in nul1 detector. Care was taken to avoid heating effects by keeping the measuring current sufficiently low $(\sim 1 \mu \mathrm{A})$. Soft gold-plated spring contacts were used as electrodes. A magnetic field could be applied to the samples both parallel to the direction of the average current flow (longitudinal MR) and perpendicular (transverse MR). In addition a Corbino geometry was employed. In this case the concentric electrodes were painted directly on the sample (gold paint). We did not notice any significant influence of the particular geometry on our data.

Upon coo1-down the resistance $R_{0}$ of our samples (measured in zero magnetic field) increased at a rate which depended very much on the doping level. Whereas high-Ohmic samples 
(specific resistance $\rho$ at $4.2 \mathrm{~K} \gtrsim 10^{-3} \Omega \mathrm{m}$ ) showed the well-known exp $\left(\mathrm{T}_{\mathrm{o}} / \mathrm{T}\right)^{\mathrm{I} / 4}$ behavior characteristic for "variable-range hopping" (VRH), the more conducting samples ( $\leqslant \leqslant 10^{-3} \Omega$ an at $4.2 \mathrm{~K}$ ) had much less variation, sometimes following a power law $R_{0} \propto T^{-x}$ with $x<1$, though no consistent picture could be obtained in the latter case. Fig. 1 shows some typical temperature dependencies obtained in zero magnetic field. The different

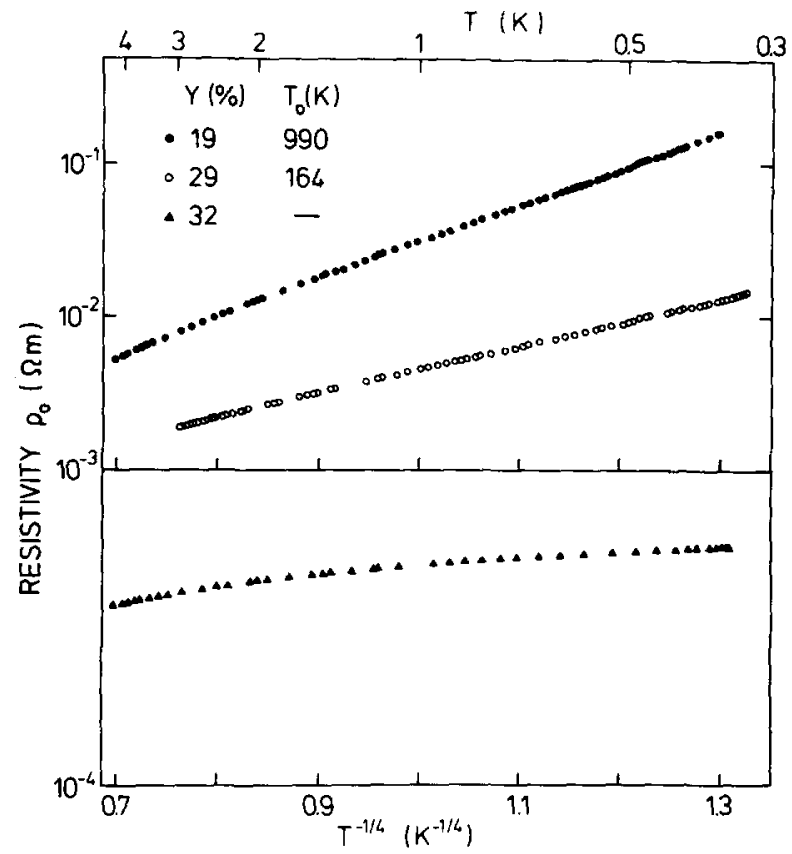

Fig. 1. Temperature dependence of the resistance of various samples of $\left(\mathrm{CHI}_{y}\right)_{x}$ in $\mathrm{B}=0$. The values for $T_{0}$ are from a fit to $a \exp \left(T_{0} / T\right)^{1 / 4}$ law. The scale for the lowest curve has been expanded in order to demonstrate the different functional form.

behavior of the more metallic samples is probably due to some other conduction process, e.g. impurity band conduction. In fact, it is somewhat surprising that even at doping levels as high as about $20 \%$ we still observe VRH though the mean distance between the acceptor sites (I $\overline{3})$ could be as short as $10 \AA$. It should be mentioned that most of the data are also consistent with somewhat different dependencies like $\exp \left(\mathrm{T}_{\mathrm{O}} / \mathrm{T}\right)^{1 / \mathrm{n}}$ with $\mathrm{n}$ ranging from 2 to 5 . Because these temperature dependencies are fairly weak it is impossible to determine $n$ accurately from our data which cover only a rather limited interval on the $\mathrm{T}^{-1 / \mathrm{n}}$ scales. Much more detailed information, however, is generally obtained from the MR which for VRH strongly depends on both the temperature and the magnetic field.

Applying a magnetic field always resulted in an increase of the resistance (positive MR: $\left.\Delta R / R_{0} \equiv\left(R(B)-R_{0}\right) / R_{0}>0\right)$ except for the heavily doped samples at high temperatures $(\sim 4.2 \mathrm{~K})$ where it became vanishingly small $\left(\Delta R / R_{0}<10^{-4}\right)$. Figure 2 shows a characteristic set of data obtained at various constant temperatures. All of

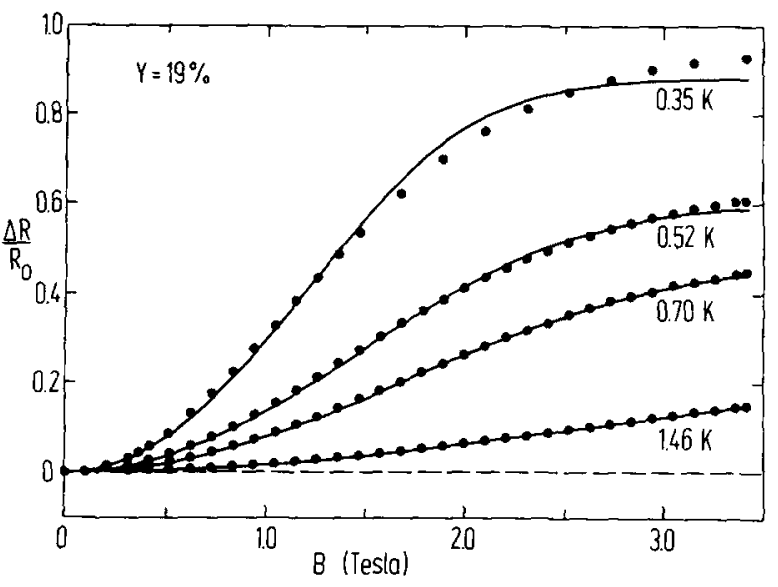

Fig. 2. Longi tudinal magnetoresistance $\Delta R / R_{O}$ at various temperatures. The solid lines are fits of equation (2).

our samples and geometries had the following behavior. The MR increased rapidly with decreasing temperature becoming very large $\left(\Delta R / R_{0}>1\right)$ at low temperatures for the more resistive samples. The more conducting samples had a much lower MR which at low temperatures saturated ${ }^{7}$ when the magnetic field was increased to $3.4 \mathrm{~T}$. Analyzing the small field behavior we find the MR to be proportional to $\mathrm{B}^{2}$ and within our temperature interval to scale as $\mathrm{T}^{-2}$ below $1 \mathrm{~K}$ and as $\mathrm{T}^{-3}$ above $2 \mathrm{~K}$, see Fig. 3. The $\mathrm{B}^{2}$
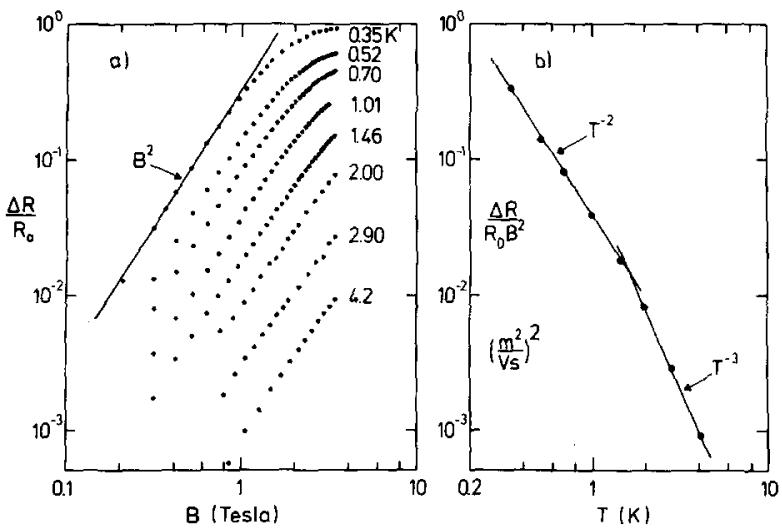

Fig. 3. a) In small fields the magnetoresistance is proportional to $\mathrm{B}^{2}$. b) The coefficient is strongly temperature dependent.

regime became narrower as the samples were doped more heavily because of the tendency of the MR to saturate at low temperatures in spite of its small value. Thus it is not the absolute value of $\Delta R / R_{0}$ which determines the deviation from the $B^{2}$ law. At $4.2 \mathrm{~K}$ the MR (when measurable at a11) remained proportional to $\mathrm{B}^{2}$ up to our highest field.

Analyzing the saturating behavior in high fields is more difficult because of the limited data. Only at low temperatures and high iodine concentrations saturation could be observed. Furthermore, this regime might be affected by 
the onset of a different MR. ${ }^{7}$ Therefore, we can make only the following qualitative remark. Saturation is approached probably not by any power law but rather exponentially fast (see below). Further work at lower temperatures and/ or higher fields is required to determine this regime unambiguously.

Turning to the interpretation of our results we feel that out of the various effects a magnetic field can have on the charge carriers the shrinking of the hole wavefunction which inhibits the hopping mechanism ${ }^{8}$ is the most likely candidate in our case. This interpretation is supported in particular by the zero field data indicating a VRH type charge transport. Furthermore, the above mentioned geometry independence of our data seem to rule out the classical MR caused by deflection of trajectories by the Lorentz force. 9 In fact, the fleecy structure of polyacetylene most certainly inhibits the buildup of a Hall voltage. 10 For the VRH mechanism at moderate fields only a small anisotropy may be expected ${ }^{8}$ which will be further reduced by the unoriented fibrillar structure.

Assuming VRH the conductivity o is reduced by a moderate magnetic field according to

$$
\sigma(B)=\sigma_{1} \exp \left(-B^{2} / B_{o}^{2}\right)
$$

In case of a constant density of states $N$ at the Fermi level $E_{F}$ one has $B_{0}^{2} \propto T^{3} / 4$. If, on the other hand, $N \propto(\Delta E)^{2}$ where $\Delta E=E-E_{F}$, one finds $B_{O}^{2} \propto T^{3 / 2}$. For stronger fields the exponent in either case becomes less field and less temperature dependent. ${ }^{8}$ Equation (1) leads to a $M R$ increasing in proportion to $B^{2}$ in small fields and then diverging exponentially. 11 our data, though, show a tendency to saturate in higher fields which is the more pronounced the higher the doping level. We interpret this as evidence for another transport mechanism which is much less sensitive to the magnetic field and which shortens out the vanishing VRH. Amending equation (1) by an additive constant $\sigma_{2}$ we have

$$
\sigma(B)=\sigma_{1} \exp \left(-B^{2} / B_{0}^{2}\right)+\sigma_{2}
$$

From equation (2) the MR can be calculated and fit to the data by adjusting the two fit parameters $\sigma_{2} / \sigma_{1}$ and $\mathrm{B}_{0}^{2}$ at a given temperature. As can be seen from Fig.2 the fit is fair though not perfect, some systematic deviation exists at the lowest temperatures where saturation is actually approached more slowly. This is most likely due to a beginning transition to the strong field case. The temperature dependence of the fit parameters is shown in Figs. $4 a$ and $4 \mathrm{~b}$. We notice that $B_{0}^{2}$ follows approximately a power law $T^{1.5}$. For $\left(B / B_{O}\right)^{2}<<1$ (i.e. $T>2 \mathrm{~K}$ ) the two parameters are correlated and only the ratio $\sigma_{1} /\left(\sigma_{1}+\sigma_{2}\right) \mathrm{B}_{0}^{2}$ can be obtained which, of course, is identical to the coefficient $\triangle R / R_{0} B^{2}$ displayed in Fig.3. Applying equation (2) to the
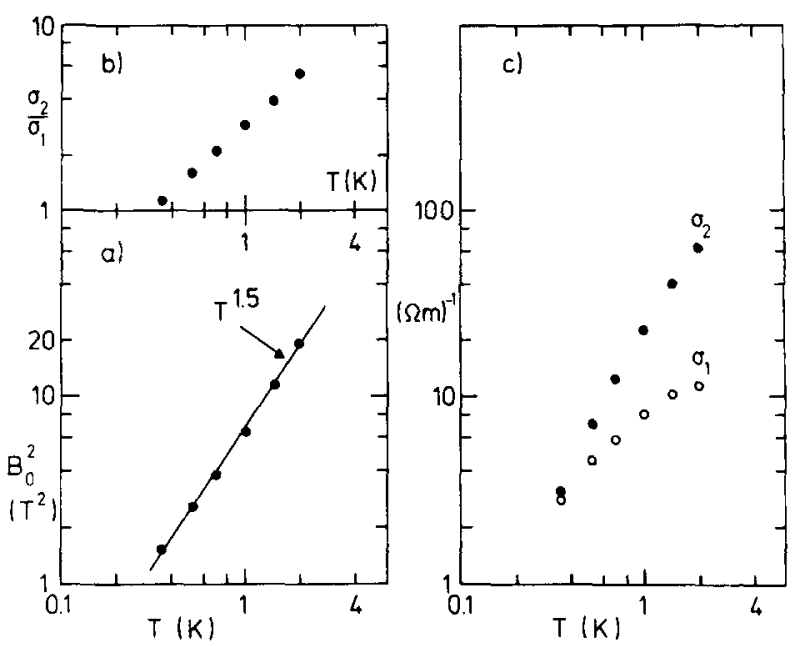

Fig. 4. Temperature dependence of the fit parameters $\mathrm{B}_{0}^{2}(4 \mathrm{a})$ and $\sigma_{2} / \sigma_{1}(4 \mathrm{~b})$ of eq. (2). 4c): $\sigma_{1}(T)$ and $\sigma_{2}(T)$ of eq. (3).

zero field data we have

$$
\rho_{0}=\sigma(o)^{-1}=\left(\sigma_{1}+\sigma_{2}\right)^{-1} \text {. }
$$

From the measured temperature dependence of $\rho_{0}$ and the known ratio $\sigma_{2} / \sigma_{1}$ (as determined above) we obtain $\sigma_{1}(T)$ and $\sigma_{2}(T)$ separately, see Fig. $4 \mathrm{c}$. In view of the crudeness of our model it is probably not very meaningful to consider these dependencies in detail, we just notice that $\sigma_{2}$ is always larger than $\sigma_{1}$ and therefore dominates the zero field data. Both quantities vary from sample to sample and temperature dependence of the small field data (Fig.3b) within this model might accidentally be given by the $T^{-2}$ and $T^{-3}$ power laws. The remarkable feature of our model is that for all samples investigated we find $\mathrm{B}_{\mathrm{o}}^{2}$ in agreement with $\mathrm{VRH}$ and $\mathrm{N} \propto(\Delta E)^{2}$. It would be desirable to have a more detailed theoretical model of the magnetoresistance to compare our data with.

In sumary, we attribute the large positive magnetoresistance we find below $4.2 \mathrm{~K}$ to a variable-range hopping conduction in the presence of another transport mechanism which is less sensitive to a magnetic field and whose nature will be investigated in more detail in the future.

Acknowledgement - We gratefully acknowledge useful discussion with W.Prett1, K.F.Renk, and G. Wegner. K.Dransfeld and K. Ehinger kindly informed us of the results of the Stuttgart and Vienna groups (ref. 4). F.Hofmann helped in running the experiment. This work was supported by the Deutsche Forschungsgemeinschaft (Regensburg) as well as by a grant from the BMFT and further support by Fonds der Chemischen Industrie (Freiburg). 


\section{References}

1. Y.-W. Park, A.J. Heeger, M.A. Druy, and A.G. MacDiarmid, J.Chem.Phys. 73, 946 (1980).

2. F.J. Kwak, T.C. Clarke, R.L. Greene, and G.B. Street, Solid State Comm. 31, 355 (1979).

3. C.M. Gould, D.M. Bates, H.M. Bozler, A.J. Heeger, M.A. Druy, and A.G. MacDiarmid, Phys.Rev. B 23, 6820 (1981).

4. W. Röss, A. Philipp, K. Seeger, K. Ehinger, K. Menke, and S. Roth, Solid State Comm. 45, 933 (1983).

5. L.B. Luttinger, J.org.Chem. 27, 1135 (1960).

6. T. Ito, H. Shirakawa, and S. Ikeda, J. Polymer Science 12, 11 (1974).

7. In our most heavily doped sample $(y=32 \%)$ at $0.33 \mathrm{~K}$ the MR clearly passed through a maximum of $7 \%$ at about 2.8 Tesla and dropped with increasing field.
8. B.I. Shklovskii, Fiz. Tekh. Poluprov. 6, 1197 (1972) [Sov.Phys.Semicond. 6, $105 \overline{3}$ (1973) ].

9. In addition, if this mechanism were the origin of the observed MR we could estimate the mobility $\mu$ from $\Delta R / R_{o} \sim(\mu B)^{2}$ giving $\mu \sim 5 \cdot 10^{3} \mathrm{~cm}^{2} /$ Vsec at $0.5 \mathrm{~K}$, a very large value for a highly disordered material like polyacetylene.

10. K. Seeger, W.D. Gill, T.C. Clarke, and G.B. Street, Solid State Comm. 28, 873 (1978).

11. K. Kahlert, G. Landwehr, A. Schlachetzki, H. Salow, Z.Physik B 24, 361 (1976). 